Adriana Aparecida Ferraz Carbonel ${ }^{1}$

Regiane Helena Barros Rabelo Santos ${ }^{2}$

Ricardo SANTOS SIMÕES ${ }^{3}$

RINALDO FLÔRENCIO SIIVA'

JOSÉ MARIA SOARES JÚNIOR ${ }^{4}$

EDMUND CHADA BARACAT ${ }^{5}$

MAURO ABI HAIDAR ${ }^{4}$

Artigo original

Palavras-chave

Glândulas mamárias animais/ efeitos de drogas

Estrogênios

Genisteína/administração \& dosagem Isoflavonas

Ratos

Keywords

Mammary glands, animal/ drug effects

Estrogens

Genistein/administration \& dosage Isoflavones

Rats

\section{Efeitos de altas doses de genisteína sobre o epitélio mamário de ratas}

\author{
Effectsofhighdosesofgenisteinonmammaryglandoffemalerat
}

\section{Resumo}

OBJETIVO: Avaliar os efeitos de altas doses de genisteína sobre o epitélio mamário de ratas adultas. MÉTODOS: Após 28 dias da ooforectomia, cinquenta ratas adultas foram divididas em cinco grupos, a saber: um controle (Ctrl), três que receberam genisteína (GEN) nas doses de 46 mg/kg (GEN46), 125 mg/kg (GEN125) e 250 mg/kg (GEN250), e um que recebeu estrogênios conjugados equinos na dose de $50 \mathrm{\mu g} / \mathrm{kg}$ (ECE). As substâncias foram administradas diariamente durante 30 dias consecutivos por gavagem e na última semana de tratamento foi efetuado exame colpocitológico durante sete dias consecutivos. Após o tratamento, os animais foram anestesiados, amostras de sangue foram retiradas para determinação do estradiol e da progesterona, e o primeiro par de mamas inguinais retirado e processado para análise histomorfométrica. Os dados obtidos foram submetidos à análise de variância complementada pelo teste de Tukey-Kramer (p<0,05). RESULTADOS: Nos grupos Ctrl e tratados com as diferentes doses de GEN as mamas apresentaram-se atróficas, no entanto mostraram-se desenvolvidas no grupo ECE, onde se notou a presença de inúmeros ductos e alvéolos mamários contendo material eosinófilo em seu interior. A morfometria mostrou maior área de parênquima mamário no grupo ECE $\left(98.870,1 \pm 550,4{\mu m^{2 *}}^{2 *}\right.$ por mm²; $\left.p<0,05\right)$ comparado aos outros

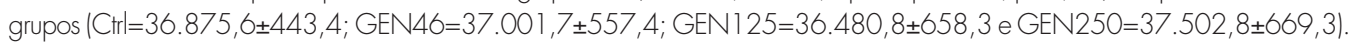
mesmo ocorreu em relação ao número de alvéolos e ductos mamários no grupo ECE $(33,2 \pm 6,9$ * por mm²; $p<0,05)$ em

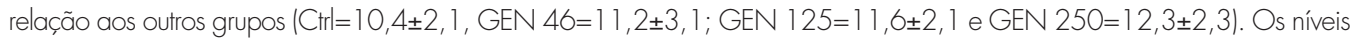
de estradiol mostraram-se aumentados no grupo ECE em relação aos outros grupos $(9,4 \pm 1,7 \mathrm{pg} / \mathrm{mL} ; \mathrm{p}<0,05)$, sendo que os níveis séricos de progesterona mostraram-se semelhantes em todos os grupos de estudo. CONCLUSÃO: A administração de genisteína em altas doses não apresentou efeito proliferativo no tecido mamário de ratas.

\section{Abstract}

PURPOSE: To evaluate the effects of high doses of genistein on the mammary glands of adult female rats. METHODS: Twenty-eight days after oophorectomy, 50 adult female rats were divided into five groups, as follows: a control group (Ctrl), three rats that received genistein (GEN) at the doses of $46 \mathrm{mg} / \mathrm{kg}$ (GEN46;), $125 \mathrm{mg} / \mathrm{kg}$ (GEN125) and $250 \mathrm{mg} / \mathrm{kg}$ (GEN250); one group received conjugated equine estrogen at the dose of $50 \mathrm{\mu g} / \mathrm{g}$ (ECE50). The substances were administered daily for 30 consecutive days by gavage and in the last week of the period of treatment, colpocytological exams were carried out for seven consecutive days. After treatment, the animals were anesthetized, blood samples were collected for estradiol and progesterone determination and the first pair of inguinal mammary glands was removed and processed for histomorphometric analysis. Collected data were subjected to analysis of variance supplemented by the Tukey-Kramer test ( $p<0.05)$. RESULTS: The Ctrl group and the ones treated with different doses of GEN showed atrophic mammary glands, whereas the glands were more developed in the ECE group, where numerous mammary ducts and alveoli were observed. Morphometry showed a larger area of mammary parenchyma in the ECE group $\left(98.870 .1 \pm 550.4 \mu^{2 *}\right.$ per $\left.\mathrm{mm}^{2} ; \mathrm{p}<0.05\right)$ compared with other groups $\mid \mathrm{Ctrl}=36.875 .6 \pm 443.4 ; \mathrm{GEN46}=37.001 .7 \pm 557.4 ; \mathrm{GEN125}=36.480 .8 \pm 658.3$ and GEN250=37.502.8 \pm 669.31 . The same occurred in the number of alveoli in the ECE group $\left(33.2 \pm 6.9^{*}\right.$ per $\left.\mathrm{mm}^{2} ; \mathrm{p}<0.05\right)$ compared to the other groups $\mid \mathrm{Ctrl}=10.4 \pm 2.1, \mathrm{GEN} 46=11.2 \pm 3.1 ; \mathrm{GEN} 125=11.6 \pm 2.1$ and $\mathrm{GEN} 250=12.3 \pm 2.3 \mid$. The estradiol level was higher in the ECE group compared to the other groups $(9.4 \pm 1.7 \mathrm{pg} / \mathrm{mL} ; \mathrm{p}<0.05)$, whereas serum levels of progesterone were similar in all groups. CONCLUSION: The administration of genistein at high doses had no trophic effect on the mammary glands of rats.
Correspondência: Adriana Aparecida Ferraz Carbone Disciplina de Histologia e Biologia Estrutural Rua Botucatu, 740 - Vila Clementino CEP: 04023-900

São Paulo (SP), Brasil

Recebido 05/09/2011

Aceito com modificações 25/09/2011
Departamento de Morfologia e Genética da Escola Paulista de Medicina da Universidade Federal de São Paulo - UNIFESP São Paulo (SP), Brasil; Departamento de Ginecologia da Escola Paulista de Medicina da Universidade Federal de São Paulo UNIFESP - São Paulo (SP), Brasil.

' Pós-Graduando do Departamento de Morfologia e Genética da Escola Paulista de Medicina da Universidade Federal de São Paulo - UNIFESP - São Paulo (SP), Brasil.

2 Pós-Graduanda do Departamento de Ginecologia da Escola Paulista de Medicina da Universidade Federal de São Paulo - UNIFESP - São Paulo (SP), Brasil.

${ }^{3}$ Pós-Graduando do Departamento de Obstetrícia e Ginecologia da Faculdade de Medicina da Universidade de São Paulo - USP São Paulo (SP), Brasil.

4 Professor Associado do Departamento de Ginecologia da Escola Paulista de Medicina da Universidade Federal de São Paulo UNIFESP - São Paulo (SP), Brasil.

5 Professor Titular do Departamento de Obstetrícia e Ginecologia da Faculdade de Medicina da Universidade de São Paulo - USP São Paulo (SP), Brasil.

Conflito de interesses: não há 


\section{Introdução}

A atrofia do sistema genital feminino é uma condição frequente na pós-menopausa, devido à queda dos hormônios ovarianos, em especial dos estrogênios ${ }^{1,2}$. Além disso, o hipoestrogenismo pode causar diversos distúrbios, tais como sintomas vasomotores, doenças cardiovasculares, osteoporose, distúrbios cognitivos, entre outros ${ }^{3}$. A terapia hormonal (TH) é um dos tratamentos alternativos para o alívio dos sintomas na pós-menopausa. No entanto, há relatos de ocorrência de efeitos adversos a longo prazo com o emprego da terapia estroprogestativa, em especial quanto ao aumento do risco de câncer mamário ${ }^{4}$. De fato, vários estudos epidemiológicos têm relatado risco aumentado de câncer de mama, sendo o risco maior com o uso de estrogênios associados aos progestagênios do que com estrogênios isolados 5 .

A mama é uma estrutura que apresenta respostas variáveis aos hormônios esteroides, sendo sua interação hormonal complexa, podendo atuar por via autócrina, parácrina e endócrina. Sob ação dos hormônios esteroides, o tecido mamário atinge seu desenvolvimento máximo ${ }^{6}$. Há evidências de que os estrogênios estimulam o crescimento ductal ${ }^{7,8}$. No entanto, para alguns, os estrogênios produzem atrofia do epitélio mamário?.

Nos últimos anos os fitoestrogênios, em especial as isoflavonas, têm se tornado atraentes como possível alternativa, por serem consideradas mais seguras devido ao fato de serem substâncias agonistas dos estrogênios e apresentarem fraca atividade estrogênica ${ }^{10}$.

As isoflavonas, os fitoestrogênios mais estudados e os mais comuns na dieta humana são encontrados principalmente em derivados da soja, tendo como principais elementos ativos a genisteína, daidzeína, biochanina $\mathrm{A}$ e formononetina ${ }^{11}$, sendo a genisteína o fitoestrogênio mais ativo e abundante da soja ${ }^{12}$. Sua eficácia tem sido investigada em inúmeros ensaios clínicos, sendo que alguns estudos referem haver redução dos sintomas vasomotores na menopausa ${ }^{13,14}$. No entanto, a maioria dos estudos refere que a administração desse composto não afeta o epitélio vaginal e nem reverte os sintomas vasculares $^{15-17}$.

Estudos epidemiológicos têm mostrado que os fitoestrogênios estão associados a uma redução no risco de câncer estrógeno-dependente, como câncer de mama, mas os efeitos sobre a prevenção do câncer ainda são controversos ${ }^{12,18-23}$. Estudos in vitro mostraram que os fitoestrogênios, em especial a genisteína, induzem apoptose em tumores mamários com células positivas aos receptores de estrogênio ${ }^{24-27}$. No campo experimental, in vivo, notamos haver inúmeros trabalhos, em especial em ratas, mostrando haver ação trófica das isoflavonas sobre o sistema genital feminino, e ação inibitória no epitélio mamário, no entanto, temos dificuldades em averiguar como se comporta a mama ante a altas dosagens ${ }^{28-31}$. Assim, propusemonos a estudar a ação da adminstração de genisteína em altas doses sobre a mama de ratas.

\section{Métodos}

Trabalho experimental, prospectivo, duplo-cego, randomizado, no qual foram usadas ratas (Rattus norvegicus albinus) adultas, virgens, pesando aproximadamente 250 gramas, fornecidas pelo Centro de Desenvolvimento de Modelos de Experimentação (CEDEME) da Universidade Federal de São Paulo Escola Paulista de Medicina (UNIFESP/EPM). Este estudo foi aprovado pelo Comitê de Ética em Pesquisa da UNIFESP/EPM (parecer $n^{\circ}$ 0749/07).

Após 28 dias da ooforectomia, 50 animas foram randomicamente divididos em cinco grupos, a saber: $\mathrm{Ctrl}$ (controle) - recebeu propilenoglicol; GEN46 - recebeu $46 \mathrm{mg} / \mathrm{kg}$, por dia de genisteína; GEN125 - que recebeu $125 \mathrm{mg} / \mathrm{kg}$, por dia de genisteína, GEN250 - recebeu $250 \mathrm{mg} / \mathrm{kg}$ de genisteína por dia e ECE - recebeu $50 \mu \mathrm{g} / \mathrm{kg}$ de estrogênios conjugados equinos. As substâncias foram diluídas em 1,0 mL de propilenoglicol, e administradas diariamente, no início do fotoperíodo claro, em torno das sete horas, por um período de 30 dias consecutivos, por gavagem.

$\mathrm{Na}$ última semana do experimento foram realizados exames colpocitológicos diários, corados pelo método de Harris-Shorr, para averiguar o estímulo do fito-hormônio. Para tanto, foi introduzida no óstio da vagina uma haste de algodão (cotonete ${ }^{\circledR}$ ) embebida em soro fisiológico, de onde se retirou material do lúmen vaginal, o qual foi depositado sobre lâmina histológica, que foi rapidamente mergulhada em solução de etanol e éter (1:1) durante 10 minutos. Ao final desse período o material foi corado pelo método de Harris-Shorr.

Após 30 dias do início da ministração dos fármacos, todos os animais foram anestesiados com xilazina $(20 \mathrm{mg} / \mathrm{kg})$ e cetamina $(100 \mathrm{mg} / \mathrm{kg})$, por via intraperitoneal, sendo retirados $2 \mathrm{~mL}$ de sangue do ventrículo esquerdo e a seguir o primeiro par de glândulas mamárias inguinais foi localizado, procedendo-se então a sua retira$\mathrm{da}$, sendo os animais eutanasiados pelo aprofundamento do plano anestésico.

Após a sua retirada, as mamas foram mergulhadas em formol a $10 \%$ e, após a fixação, as amostras, desidratadas em concentrações crescentes de álcool etílico, diafanizadas em xilol e impregnadas e incluídas em parafina. Foram obtidos cortes $(5 \mu \mathrm{m})$ os quais foram corados pela hematoxilina e eosina (HE). 
A avaliação morfológica foi feita pela microscopia de luz (microscópio Carl Zeiss ${ }^{\circledR}$ ), com oculares de 10 vezes e objetivas com aumento de 10 e 40 vezes. A morfometria foi realizada por digitalização de imagens com o programa AxioVision 4.1 $\left(\right.$ Carl Zeiss $\left.^{\circledR}\right)$. Para tanto, foi delimitada a área ocupada pelo parênquima mamário em uma área de $60 \times 10^{4} \mu \mathrm{m}^{2}$ em cada corte. Foram avaliados dez cortes semisseriados em cada animal do experimento, sendo avaliada uma área total $60 \mathrm{~mm}^{2}$ em cada grupo de estudo.

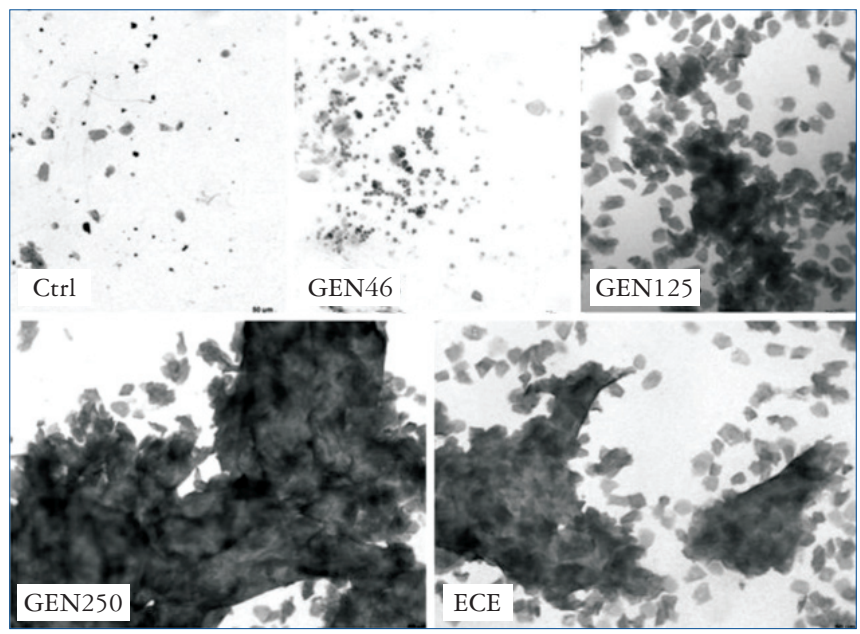

Figura 1. Fotomicrografias mostrando colpocitológicos de ratas ovariectomizadas (Ctrl), tratadas com diferentes concentrações de genisteína (GEN46=46 mg/kg; GEN125=125 mg/kg; GEN=250 mg/kg) e estrogênios conjugados equinos (ECE $=50 \mu \mathrm{g} / \mathrm{kg})$. No Ctrl e GEN46, notar escassez de células oriundas da descamação do epitélio vaginal e inúmeros leucócitos. Em GEN125 e GEN250, observar células epiteliais anucleadas e acidófilas oriundas das camadas mais superficiais do epitélio vaginal, e em ECE, grande concentração de células superficiais. Harris.Shorr - $100 \mathrm{X}$

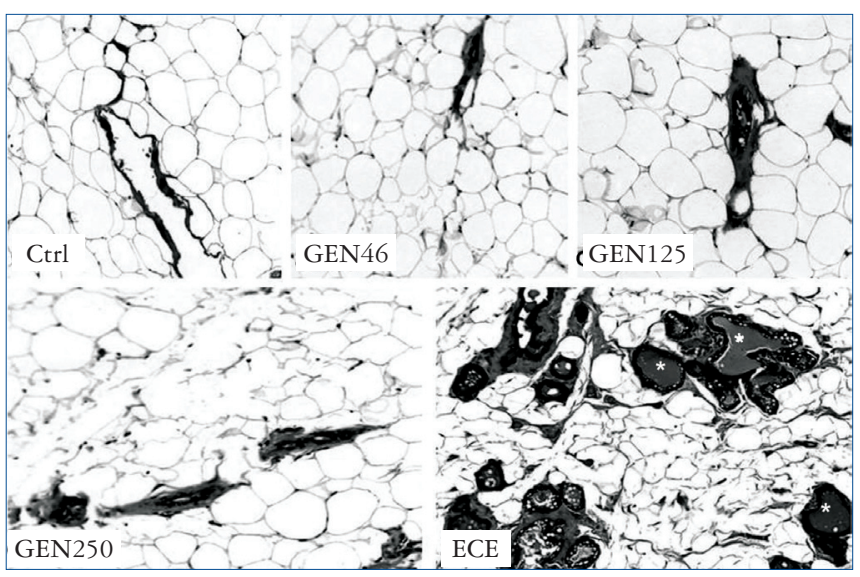

Figura 2. Fotomicrografias mostrando porção de tecido mamário de ratas pertencentes aos vários grupos de estudo. Ctrl=controle; tratados com diferentes doses de genisteína (GEN46=46mg/kg; GEN125=125 mg/kg; GEN250=250 mg/kg) e com estrogênios conjugados equinos ( $E C E=50 \mu \mathrm{g} / \mathrm{kg}$ ). Notar ductos mamários (setas) e alvéolos (*). H.E - $400 \mathrm{X}$
Os níveis séricos de $17 \beta$-estradiol $\left(\mathrm{E}_{2}\right)$ e de progesterona $\left(\mathrm{P}_{4}\right)$ foram avaliados. Após a coleta, amostras de sangue foram imediatamente centrifugadas a $4{ }^{\circ} \mathrm{C}$ $(1500 \times \mathrm{g})$ por 10 minutos e o sobrenadante congelado a $-20{ }^{\circ} \mathrm{C}$ para posterior análise por radioimunoensaio (RIA), utilizando a técnica de duplo anticorpo em tubos pré-revestidos (ICN Biomedical Inc. Costa Mera, CA, EUA), de acordo com as instruções do fabricante. Os ensaios foram realizados em duplicata. As amostras foram processadas no mesmo dia. Os limites de detecção para a determinação de $\mathrm{E}_{2}$ e $\mathrm{P}_{4}$ foram, em média, $0,1 \mathrm{pg} / \mathrm{mL}$ e $0,1 \mathrm{ng} / \mathrm{mL}$, respectivamente.

Os dados obtidos foram submetidos à análise de variância (ANOVA) complementada pelo teste de Tukey-Kramer para múltiplas comparações. O nível de significância da hipótese nula foi fixado em $5 \%(\mathrm{p}<0,05)$.

\section{Resultados}

A colpocitologia mostrou nos animais pertencentes aos grupos Ctrl e GEN46 poucos elementos epiteliais e leucócitos frequentes (Figura 1). Já nos grupos GEN125, GEN250 e ECE, o exame colpocitológico mostrou grande concentração de células acidófilas, anucleadas (Figura 2).

Os níveis séricos de progesterona $\left(\mathrm{P}_{4}\right)$ mostraram-se semelhantes em todos os grupos estudados, no entanto o nível do $17 \beta$-estradiol $\left(\mathrm{E}_{2}\right)$ mostrou-se significantemente elevado no grupo ECE $(9,4 \pm 1,7 \mathrm{pg} / \mathrm{mL}, \mathrm{p}<0,05)$ em relação aos demais.

As mamas dos animas pertencentes aos grupos Ctrl, GEN46, GEN125 e GEN250 estavam representadas por ductos e raros alvéolos mamários imersos em grande concentração de tecido adiposo. Os ductos mamários apresentaram-se constituídos por duas ou mais camadas de células epiteliais rodeadas por fibras colágenas (Figura 1). Em alguns locais pôde-se notar a presença de alvéolos mamários, os quais são formados por apenas uma camada de células epiteliais. Já no grupo ECE notou-se que o parênquima mamário estava constituído por inúmeros ductos e alvéolos mamários bem desenvolvidos, a maioria contendo material eosinófilo no seu interior (Figura 1).

A morfometria da mama não se mostrou diferente quanto à concentração de parênquima mamário e no número de ductos e alvéolos entre os vários grupos tratados com diferentes doses de genisteína e o controle, sendo maior a quantidade e a porcentagem de área ocupada pelo parênquima mamário, assim como de ductos e alvéolos no grupo ECE em relação aos demais grupos $(\mathrm{ECE}>\mathrm{GEN} 250=\mathrm{GEN} 125=\mathrm{GEN} 46=\mathrm{GCtrl}$; $\mathrm{p}<0,05)($ Tabela 1). 
Tabela 1. Dosagens séricas de estradiol, progesterona e morfometria da mama de ratas tratadas com diferentes doses de genisteína e estrogênios conjugados equinos

\begin{tabular}{|c|c|c|c|c|c|}
\hline & \multicolumn{5}{|c|}{ Grupos de estudo } \\
\hline & Ctrl & GEN46 & GEN125 & GEN250 & ECE \\
\hline \multicolumn{6}{|l|}{ Dosagem hormonal } \\
\hline Estradiol (pg/mL) & $2,5 \pm 0,4$ & $3,0 \pm 0,6$ & $2,8 \pm 1,3$ & $3,4 \pm 0,8$ & $9,4 \pm 1,7^{\star}$ \\
\hline Progesterona (ng/mL) & $1,4 \pm 0,3$ & $1,6 \pm 0,9$ & $1,5 \pm 0,8$ & $1,3 \pm 0,7$ & $1,4 \pm 0,5$ \\
\hline \multicolumn{6}{|l|}{ Mama } \\
\hline Área ocupada pelo parênquima mamário por campo ( $p \mathrm{~m}^{2}$ ) & $36.875,6 \pm 443,4$ & $37.001,7 \pm 557,4$ & $36.480,8 \pm 658,3$ & $37.502,8 \pm 669,3$ & $98.870,1 \pm 550,4^{*}$ \\
\hline Número de ductos mamários e alvéolos $/ \mathrm{mm}^{2}$ & $10,4 \pm 2,1$ & $11,2 \pm 3,1$ & $11,6 \pm 2,1$ & $12,3 \pm 2,3$ & $33,2 \pm 6,9^{\star}$ \\
\hline
\end{tabular}

Ctrl: grupo controle; GEN46: recebeu 46mg/kg de GEN; GEN125: recebeu $125 \mathrm{mg} / \mathrm{kg}$ de GEN; GEN250: que recebeu $250 \mathrm{mg} / \mathrm{kg}$ de GEN; ECE: recebeu 50 pg/kg de ECE; $\mathrm{p}<0,05$

\section{Discussão}

Alguns autores referem que as isoflavonas, nas doses normalmente utilizadas não melhoram os sintomas vasomotores da menopausa ${ }^{15-17}$, havendo melhora desses sintomas com o uso de altas doses ${ }^{14}$. Assim, o presente estudo teve como objetivo averiguar a morfologia da mama de ratas ante a altas concentrações de isoflavonas, em especial da genisteína.

As isoflavonas correspondem à mistura de substâncias com diferentes afinidades pelos receptores de estrogênio. Dentre estas, destaca-se a genisteína, que apresenta maior atividade estrogênica. Em trabalho anterior utilizando o mesmo tipo de isoflavonas determinou-se que a dose de $125 \mathrm{mg} / \mathrm{kg}$ foi suficiente para aumentar significantemente o peso do útero em ratas castradas e de induzir queratinização vaginal ${ }^{32}$. Assim, escolhemos como alta dose de isoflavonas o dobro da dose mínima de genisteína capaz de produzir em comparação ao grupo controle, o que corresponde à dose de $250 \mathrm{mg} / \mathrm{kg}^{32}$. Como controle positivo utilizamos estrogênios conjugados equinos. Deve ainda ser ressaltado que trabalho anterior mostrou que essa mesma dose de genesteína induz metaplasia escamosa nos epitélios luminal e glandular do endométrio de ratas, daí ser considerada por nós como alta dose $e^{33}$.

Em nosso experimento notamos haver efeitos tróficos no trato genital das ratas nos grupos tratados com 125 e $250 \mathrm{mg} / \mathrm{kg}$ de genisteína, uma vez que encontramos grande concentração de células anucleadas e acidófilas no esfregaço vaginal. Já o grupo tratado com $46 \mathrm{mg} / \mathrm{kg}$ não apresentou nenhum efeito, servindo assim como controle negativo. Tivemos ainda o cuidado de realizar dosagens séricas dos estrogênios $\left(\mathrm{E}_{2}\right)$ e da progesterona $\left(\mathrm{P}_{4}\right)$ para descartar uma possível fonte endógena desses hormônios, e assim poder inferir que os dados obtidos na colpocitologia, assim como na mama são devidos exclusivamente à genisteína ou à administração dos estrogênios conjugados equinos. As dosagens hormonais mostraram níveis séricos do $\mathrm{E}_{2}$ e da $\mathrm{P}_{4}$ baixos e semelhantes em todos os grupos tratados com isoflavonas, estando o $\mathrm{E}_{2}$ aumentado no grupo ECE.
Com relação à mama, nosso estudo mostrou que a genisteína não apresentou efeitos tróficos, o que pode ser notado pelos aspectos morfológicos, assim como da área ocupada pelo parênquima mamário e no número de ductos e alvéolos $/ \mathrm{mm}^{2}$, quando os animais foram tratados pelo período de 30 dias consecutivos. Deve ser ressaltado que os compostos de isoflavonas têm, além da genisteína, outras substâncias que, por terem semelhança estrutural e funcional com os estrogênios, podem modular os receptores de estrogênio. Já o tratamento com ECE mostrou ter efeito trófico sobre a mama de ratas.

Estudos de cultura de células mamárias relataram propriedades antiproliferativas da genisteína, que atuaria como um modulador seletivo do receptor de estrogênio beta $(\operatorname{ER} \beta)$, bem como ação antioxidante, modulação da apoptose, inibição da topoisomerase II, do fator de crescimento tumoral beta 1 e do fator de crescimento epidérmico ${ }^{34,35}$. Estudos relatam que dieta baseada em soja inibiria o crescimento do câncer mamário induzido por carcinogênicos ${ }^{24,36,37}$.

O mecanismo de ação das isoflavonas no tecido alvo resulta, entre outros, em efeitos estrogênicos ou antiestrogênicos dependendo do tipo e concentração de receptor estimulado nos tecidos e da sua concentração no organismo ${ }^{38}$. Atualmente as isoflavonas são consideradas moduladores seletivos naturais do receptor de estrogênio (SERMs) com atividade estrogênica e antiestrogênica ${ }^{11,35}$.

Assim, a genisteína mostra ter maior afinidade para receptores de estrógeno beta $(\mathrm{ER} \beta)^{34}$, mais frequentes na vagina e útero e menos frequentes no tecido mamário, que é rico em receptores de estrogênio alfa $(\mathrm{ER} \alpha)^{39}$, o que poderia ser uma das possíveis explicações de não termos observado em nosso estudo efeito trófico das isoflavonas no tecido mamário. Os receptores de estrógeno beta apresentam ligação de 500 a 1000 vezes mais fraca aos estrogênios endógenos do que os receptores alfa, no entanto as isoflavonas podem produzir os mesmos níveis de bioatividade do estradiol, desde que em altas concentrações, o suficiente para atingir o máximo de resposta, indicando que o receptor de estrogênio 
e os complexos receptor-isoflavonoide podem ser funcionalmente equivalentes ${ }^{40}$. Assim, doses elevadas poderiam ser benéficas no tratamento dos sintomas vasomotores em mulheres na pós-menopausa, sem apresentar efeito trófico na mama, no entanto, não devem ser recomendadas devido aos efeitos uterinos.

Além disso, Sacks et al. ${ }^{41}$ sugerem que tanto o uso de suplementos de isoflavonas nos alimentos como em pílulas não devem ser recomendados; pois muitos produtos de soja considerados benéficos para a saúde cardiovascular teriam suas ações baseadas no alto conteúdo de gorduras poliinsaturadas, fibras, vitaminas, minerais e baixo conteúdo de gordura saturada e não no conteúdo de genisteína.

$\mathrm{Na}$ prática, a relação risco-benefício dos fitoestrogênios no uso clínico precisa ser devidamente avaliada, especialmente considerando que uma ingestão elevada na concentração de genisteína pode ter efeito adverso sobre o endométrio. Portanto, dados clínicos mais sólidos devem ser obtidos antes de se receitar o consumo de altas doses de fitoestrogênios para mulheres, pois estas substâncias podem ser potencialmente perigosas na medida em que induzem a proliferação e metaplasia do endométrio.

\section{Agradecimentos}

Ao Professor Manuel de Jesus Simões do Departamento de Morfologia e Genética da Universidade Federal de São Paulo - UNIFESP.

À Coordenação de aperfeiçoamento de Pessoal de Nível Superior - Capes; Conselho Nacional de Desenvolvimento Científico e Tecnológico - $\mathrm{CNPq}$ - fontes de financiamento do projeto.

\section{Referências}

1. Pastore LM, Carter RA, Hulka BS, Wells E. Self-reported urogenital symptoms in postmenopausal women: women's health initiative. Maturitas. 2004;49(4):292-303

2. Asociación Mexicana para el estudio del Climaterio. Study and treatment of women in the climacteric and postmenopause. Ginecol Obstet Mex. 2010;78(8):423-40.

3. Cavadas LF, Nunes A, Pinheiro M, Silva PT. Management of menopause in primary health care. Acta Med Port. 2010;23(2):227-36.

4. Kerlikowske K, Cook AJ, Buist DS, Cummings SR, Vachon C, Vacek $P$, et al. Breast cancer risk by breast density, menopause, and postmenopausal hormone therapy use. J Clin Oncol. 2010;28(24):3830-7.

5. Santen R, Allred DC, Ardoin SP, Archer DF, Boyd N, Braunstein GD, et al. Postmenopausal hormone therapy: an Endocrine Society scientific statement. J Clin Endocrinol Metab. 2010;95(7 Suppl 1):s 1-s66

6. Nair R, Junankar S, O'Toole S, Shah J, Borowsky AD, Bishop $J M$, et al. Redefining the expression and function of the inhibitor of differentiation 1 in mammary gland development. PLoS One. 2010;5(8):e 11947

7. Mauvais-Jarvis P, Kuttenn F, Gompel A. Estradiol/progesterone interaction in normal and pathologic breast cells. Ann N Y Acad Sci. 1986;464:152-67.

8. Chang KJ, Lee TT, Linares-Cruz G, Fournier S, de Ligniéres B. Influences of percutaneous administration of estradiol and progesterone on human breast epithelial cell cycle in vivo. Fertil Steril. 1995;63(4):785-91.

9. Silva BB, Gebrim LH, Simões M, Baracat EC, Lima GR. Efeitos do tamoxifeno e dos estrogênios conjugados no epitélio mamário de ratas em estro permanente. Rev Bras Ginecol Obstet. 2000;22(1):33-6.

10. Molla MD, Hidalgo-Mora JJ, Soteras MG. Phytotherapy as alternative to hormone replacement therapy. Front Biosci (Schol Ed). $2011 ; 3(1): 191-204$.

11. Setchell KD. Soy isoflavones-benefits and risks from nature's seletive estrogen receptor modulators (SERMs). J Am Coll Nutr. 2001;2015 Suppl):354S-62.
12. Pavese JM, Farmer RL, Bergan RC. Inhibition of cancer cell invasion and metastasis by genistein. Cancer Metastasis Rev. 2010;29(3):465-82

13. Faure ED, Chantre P, Mares P. Effects of a standardized soy extract on hot flushes: a multicenter, double blind, randomized, placebocontrolled study. Menopause. 2002;9(5):329-34.

14. Ferrari A. Soy extract phytoestrogens with high dose of isoflavones for menopausal symptoms. J Obstet Gynaecol Res. 2009;35(6): 1083-90.

15. Nikander E, Rutanen EM, Nieminen P, Wahlström T, Ylikorkala $O$, Tiitinen A. Lack of effect of isoflavonoids on the vagina and endometrium in postmenopausal women. Fertil Steril. 2005;83(1): 137-42.

16. Fernandes LFC, Aldrighi APS, Aldrighi JM. Prescrever ou não isoflavonas de soja à mulher no climatério? Rev Assoc Med Bras. 2006;52(3): 132 .

17. Villa $P$, Costantini $B$, Suriano R, Perri C, Macrì F, Ricciardi $L$, et al. The differential effect of the phytoestrogen genistein on cardiovascular risk factors in postmenopausal women: relationship with the metabolic status. J Clin Endocrinol Metab. 2009;94(2):552-8.

18. Dixon RA. Phytoestrogens. Annu Rev Plant Biol. 2004;55:225-61.

19. Magee PJ, Rowland IR. Phyto-oestrogens, their mechanism of action: current evidence for a role in breast and prostate cancer. Br J Nutr. 2004;91 (4):513-51.

20. Trock BJ, Hilakivi-Clarke L, Clarke R. Meta-analysis of soy intake and breast cancer risk. J Natl Cancer Inst. 2006;98(7):459-71.

21. Butler LM, Wu AH, Wang R, Koh WP, Yuan JM, Yu MC. A vegetable-fruit-soy dietary pattern protects against breast cancer among postmenopausal Singapore Chinese women. Am J Clin Nutr. 2010;91(4):1013-9.

22. Thompson DD, Simmons HA, Pirie CM, Ke HZ. FDA guidelines and animal models for osteoporosis. Bone. 1995;17/4 Suppl):S125-S33.

23. Palacios S, Pornel B, Vázquez F, Aubert L, Chantre P, Marès P. Long-term endometrial and breast safety of a specific, standardized soy extract. Climacteric. 2010;13(4):368-75. 
24. Rice S, Whitehead SA. Phytoestrogens and breast cancer - promoters or protectors? Endocr Relat Cancer. 2006;13(4):995-1015.

25. Lavigne JA, Takahashi Y, Chandramouli GV, Liu H, Perkins SN, Hursting SD, et al. Concentration-dependent effects of genistein on global gene expression in MCF-7 breast cancer cells: an oligo microarray study. Breast Cancer Res Treat. 2008;1 10(1):85-98.

26. Kim SH, Kim SH, Lee SC, Song YS. Involvement of both extrinsic and intrinsic apoptotic pathways in apoptosis induced by genistein in human cervical cancer cells. Ann N Y Acad Sci. 2009;1171:196-201.

27. Sakamoto T, Horiguchi H, Oguma E, Kayama F. Effects of diverse dietary phytoestrogens on cell growth, cell cycle and apoptosis in estrogen-receptor-positive breast cancer cells. J Nutr Biochem. 2010;21 (9):856-64.

28. Piovesan AC, Soares Júnior JM, Mosquette R, Simões $M$, Simões RS, Baracat EC. Estudo morfológico e molecular da mama de ratas castradas tratadas com isoflavona ou estrogênios. Rev Bras Ginecol Obstet. 2005;27(4):204-9.

29. Mosquette R, Simões M, Silva ID, Oshima CT, Oliveira-Filho RM, Haidar MA, et al. The effects of soy extract on the uterus of castrated adult rats. Maturitas. 2007;56(2):173-83.

30. Carbonel AAF, Haidar MA, Simões RS, Rabelo RHB, Simões $M$, Soares JM, et al. Proliferation histomorphometric and immunohistochemical markers on the ovariectomized rat vagina after estrogen and/or isoflavones treatments. Microsc Microanal. 2010;(16 Suppl 2): $1156-7$.

31. Carbonel AA, Baracat MC, Simões RS, Simões M, Baracat EC, Soares JM Jr. The soybean concentrated extract proliferates the vagina of adult rats. Menopause. 2011;18(1):93-101.

32. Santos ET, Sampaio MD, Cecon PR, Simões M, Sartori MG, Girão M. Effects of soy isoflavones on the uterus and urethra of ovariectomized rats. Int Urogynecol J. 2010;21(1):111-6.
33. Carbonel AAF, Simões RS, Rabelo RHB, Baracat MCP, Simões M, Baracat EC, et al. Efeitos das isoflavonas em altas doses sobre o útero da rata. Rev Assoc Med Bras. 201 1;57(5):534-9.

34. Morito K, Hirose T, Kinjo J, Hirakawa T, Okawa M, Nohara T, et al. Interaction of phytoestrogens with estrogen receptors alpha and beta. Biol Pharm Bull. 2001;24(4):351-6.

35. Siow RC, Mann GE. Dietary isoflavones and vascular protection: activation of cellular antioxidant defenses by SERMs or hormesis? Mol Aspects Med. 2010;31(6):468-77.

36. Peeters PH, Keinan-Boker L, van der Schouw YT, Grobbee DE. Phytoestrogens and breast cancer risk. Review of the epidemiological evidence. Breast Cancer Res Treat. 2003;77(2):171-83.

37. Wood CE, Register TC, Franke AA, Anthony MS, Cline JM. Dietary soy isoflavones inhibit estrogen effects in the postmenopausal breast. Cancer Res. 2006;66(2):1241-9.

38. Dornstauder E, Jisa E, Unterrieder I, Krenn L, Kubelka W Jungbaver A. Estrogenic activity of two standardized red clover extracts (Menoflavon) intended for large scale use in hormone replacement therapy. J Steroid Biochem Mol Biol. $2001 ; 78(1): 67-75$.

39. The role of isoflavones in menopausal health: consensus opinion of The North American Menopause Society. Menopause. 2000;7(4):215-29.

40. Markiewicz L, Garey J, Adlercreutz H, Gurpide E. In vitro bioassays of non-steroidal phytoestrogens. J Steroid Biochem Mol Biol. 1993;45(5):399-405.

41. Sacks FM, Lichtenstein A, Van Horn L, Harris W, Kris-Etherton P, Winston $M$, et al. Soy protein, isoflavones, and cardiovascular health: An American Heart Association Science Advisory for professionals from the Nutrition Committee. Circulation. 2006; $113(7): 1034-44$ 